\title{
DEVELOPMENT OF A LOW-COST HIGH ACCURACY PHASE SHIFTING GAUGE BLOCK INTERFEROMETER
}

\author{
KATIC, M[arko]; SIMUNOVIC, V[edran] \& BARSIC, G[orana]
}

\begin{abstract}
Prohibitive cost of available commercial gauge block interferometers resulted in numerous retrofitting projects in National Measurement Institutes, upgrading old gauge block interferometers to be used with laser sources and digital interferogram acquisition and processing. We demonstrate the possibility of building a new gauge block interferometer using mostly off-the-shelf components, with comparatively low cost and sufficiently high accuracy. Additional advantages over retrofitting are that an open platform for further research is obtained, and that reconfiguration to accommodate various measurement objects other than gauge blocks is possible. In this paper we will present the design of new phase shifting gauge block interferometer built at Croatian National Laboratory for Length, preliminary measurement results, and compare it with a retrofitted Zeiss gauge block interferometer. Keywords: gauge block calibration, Interferometry, phase shifting interferometry
\end{abstract}

\section{INTRODUCTION}

Interferometric measurement of gauge blocks is required in order to ensure traceability from the practical realization of meter, usually an Iodine stabilized HeliumNeon laser, to physical standards, usually highest class of gauge blocks. Using optical interferometry the length of physical standard is expressed in terms of wavelength of a reference laser, Figure 1. Most widely used configuration of interferometer for gauge block calibration is the Twyman-Green type (Figure 2), based on the original Michelson interferometer with a collimated beam, but other designs can be used as well. Example shown in Figure 2 is the Kosters variation of Twyman Green interferometer, which is used in Zeiss interferometer. In these kinds of interferometers, commonly known as amplitude division interferometers, the incoming beam is divided by a beamsplitter, reflected off two mirrors and then recombined after passing the beamsplitter again. These interferometers are used for calibration of gauge block length by using the gauge block wrung to a flat platen as one of the mirrors.

The quality of optical components represents the key factor of the interferometers accuracy. The incoming light needs to be expanded and collimated to provide a field of view large enough so that the entire surface of the gauge block is visible. To achieve proper collimation the source needs to be placed exactly at the focal point of the collimating lens- error in placement along the optical axis results in poor collimation, and error in radial direction results in asymmetrical collimation. Both will result in oblique illumination of the gauge block and inaccurate determination of gauge block length. Flatness of primary surface of the beamsplitter is a limiting factor for the accuracy of an interferometer, as well as the flatness of the reference mirror, sincethe deviations in reference wavefronts will be combined with actual deviation from the measured surfaces.

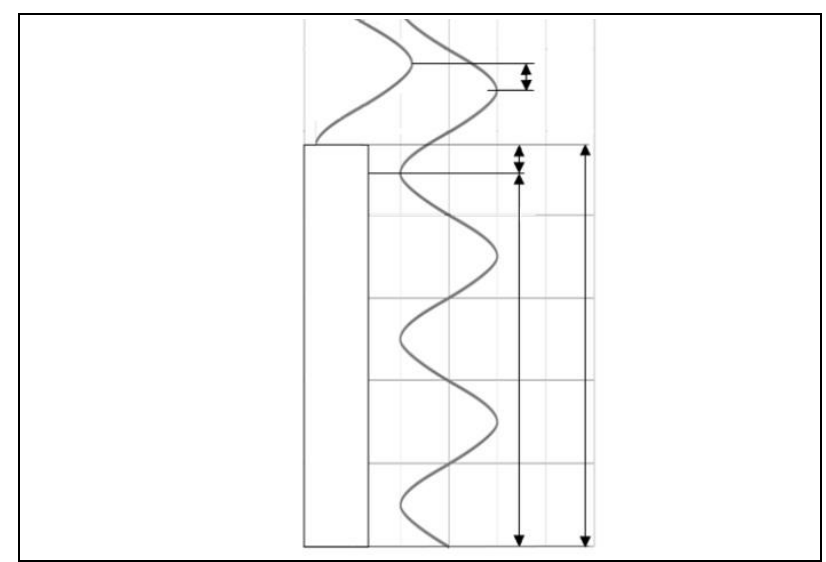

Fig. 1. Gauge block length in terms of wavelength: mechanical length is spanned by an integer number of wavelengths and remaining fraction of wavelength

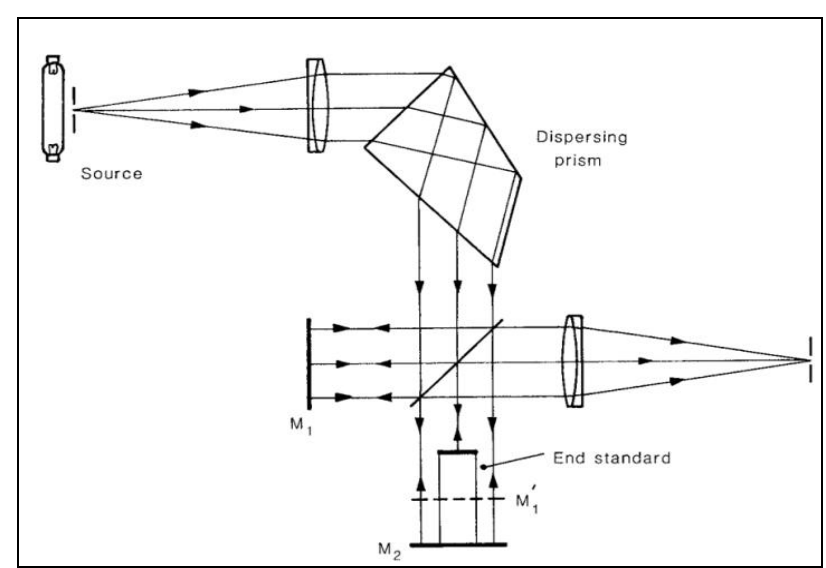

Fig. 2. Kosters gauge block interferometer design, based on the Twyman-Green type

Regardless of the type of interferometer, some common issues need to be addressed in order to achieve the required measurement uncertainties-typical expanded measurement uncertainty at European national institutes is at the level of few tens of nanometres for $100 \mathrm{~mm}$ gauge blocks.

The most important problem specific to gauge block interferometry arises from the fact that laser wavelengths are specified in vacuum. Evacuating the entire interferometer is possible, but results in an elongation of the gauge block which would have to be calculated from its modulus of elasticity E. Since this value has a very large uncertainty, measurements in vacuum are usually 
avoided. This means that the optical path of interferometer goes through air, and its index of refraction has to be determined very accurately. The modified Edlen equation [1], which is most commonly used to determine the exact index of refraction of air, requires very accurate measurement of relative humidity, pressure and temperature of air. While this does complicate the measurement systems, it still results in lower overall uncertainty than vacuum systems.

Second important issue is the temperature of the gauge block itself, which needs to be measured at millikelvin accuracies. Errors in temperature measurement directly propagate to errors in determination of length, because the results have to be expressed at standard temperature of $20^{\circ} \mathrm{C}$. An error of $10 \mathrm{mK}$ in temperature measurement of a $100 \mathrm{~mm}$ steel gauge block would result in a $\sim 10 \mathrm{~nm}$ error in length measurement, and would represent a significant systematic error.

Wringing the gauge block to the platen also requires special consideration. Since the length of the gauge block is measured as the optical path difference between its upper face and the top surface of the platen, and is in fact defined as such in the relevant international standards [2], several issues arise at this level of accuracy. Even though the wringing layer between the platen and the gauge block is included in its length, the variation of thickness of this layer must be included in the uncertainty budget. Transparent platens are usually used to ensure good and repeatable wrings by visual observation; however this results in another problem.

If the material of the gauge block and platen is not the same, different phase changes on reflection will occur. Usually the materials of gauge blocks and platen cannot be exactly the same, but using the same type of material (e.g. steel gauge block and steel platen) keeps these differences low. However, if quartz plates are used, as is frequently the case so that the quality of wringing layer can be inspected, the difference in phase change will be substantial. Quartz is a dielectric material and steel is a conductor, meaning that light will reflect from quartz with a $180^{\circ}$ phase change and from steel surface with less than $180^{\circ}$. Effectively, light is penetrating the surface of conductive materials, unlike the surface of dielectrics. Depth of penetration is typically around 30 nm.

This is the largest systematic deviation which is applied to the measured length as a correction, with amount that equals the entire uncertainty of measurement- if its value is not determined accurately the measurements can be completely inaccurate.

\section{DEVELOPMENT OF NEW PSI INTERFEROMETER AT HMI/FSB-LPMD}

The decision to build a new gauge block interferometer at HMI/FSB-LPMD was made because this approach can, at least in theory, match the accuracy of commercially available interferometers while keeping the costs low. Another way to achieve traceable gauge block calibration is retrofitting old interferometers, as described in [3], [4], [5] and [6]. Similar retrofit was done at HMI/FSB-LPMD, [7], and this system's excellent performance was successfully confirmed with a
EURAMET Key Comparison, [8]. However, building a new interferometer provides the possibility of expanding the measurement capabilities beyond measurement of gauge blocks, which is a feature that cannot be found in commercial gauge block interferometers due to their closed architecture. Also, Zeiss interferometer that was retrofitted at HMI/FSB-LPMD cannot accept multiple wavelengths simultaneously, which is a common problem for interferometers designed for use with broadband light sources and prevents the use dynamic synthetic wavelength interferometry.

The mechanical design, optical components, systems for measurement of environmental parameters, and measurement software are described in the following sections.

\subsection{Mechanical design}

The fundamental choice in designing a gauge block interferometer is determining which type of interferometer shall be used. We selected the TwymanGreen design as an optimum between achievable accuracy and number (and cost) of necessary parts.

In an effort to keep the cost as low as possible, most of mechanical components were either standard parts available off-the-shelf, or manufactured for the Laboratory. The entire setup was first modelled in CAD software, and then the deformations were calculated with FEM analysis to ensure adequate rigidity of the design. After iterative modifications of the design all deformations of the support structure was sufficiently low so that its influence could be neglected. The actual interferometer is shown in Figure 3, and Figure 4 shows a CAD model of another possible configuration as a Mach Zender interference microscope.

Optical fibre was chosen for input of laser light, so a 4f imaging system for collimation and subsequent focusing onto a precision pinhole was designed. This system provides 6 degrees of freedom with differential micrometre screws, enabling very high precision of positioning of optical fibre with respect to the collimation lens. The light is then focused onto a $10 \mu \mathrm{m}$ pinhole which serves as a spatial filter, and can also be positioned in $\mathrm{x}$ and $\mathrm{y}$ direction with micrometre accuracy. The pinhole acts as the entrance aperture, so this assembly can be precisely positioned with respect to the main collimation lens. This entire collimation assembly can also be translated vertically along the centre pole.

The beamsplitter is held in a kinematic mount with two rotational and one translational degree of freedom. It is also mounted on the centre pole, which ensures stability of its position with respect to the collimation assembly.

The holders for reference mirror and decollimation lens are kinematic mounts providing precise rotations in two axes and translations in three directions.

Phase shifting is achieved with a closed-loop piezoactuator with $0.2 \mathrm{~nm}$ repeatability, which serves as the platform for gauge block/platen assembly. The piezoactuator is mounted on a precision goniometer with 
1 arc second resolution to provide stable control of tilt, which is used to align the measurement beam.

The system described above provides enough degrees of freedom to allow accurate installation and alignment of all optical components, while providing good mechanical stability.

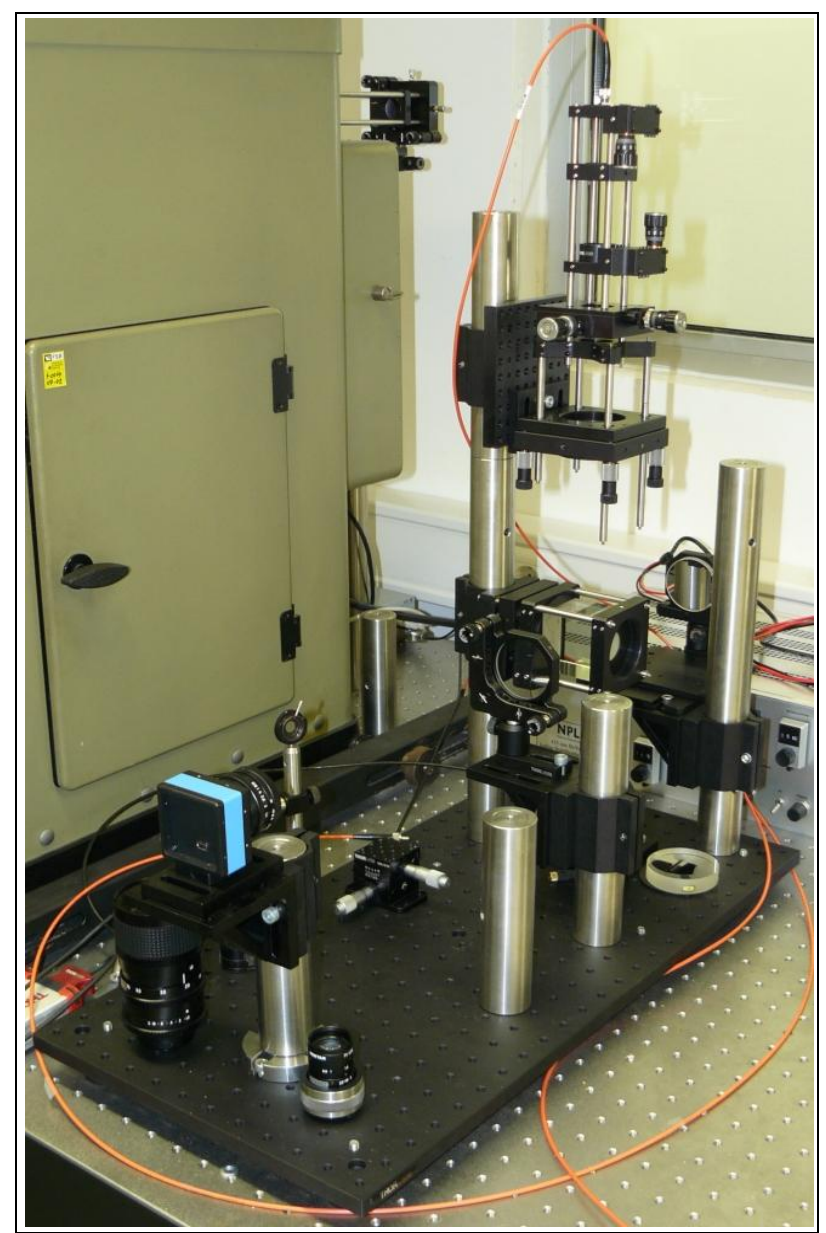

Fig. 3. New gauge block interferometer built at HMI/FSB-LPMD

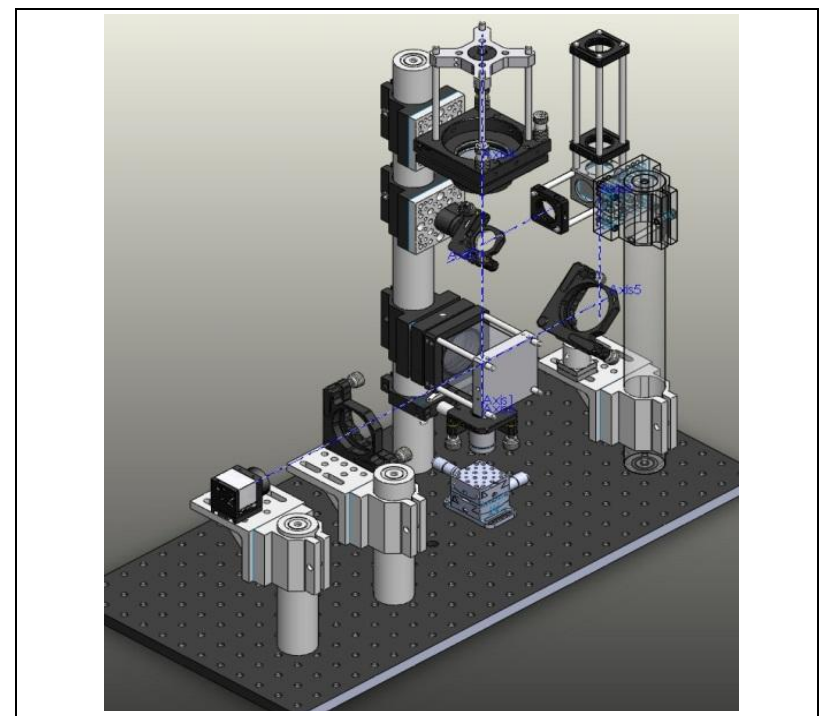

Fig. 4. CAD model of new interferometer conFig.d as a Mach Zender interferometer

\subsection{Optical components}

Typically, only highest quality optical components are installed in interferometers for gauge block calibration. The goal of this research is to determine the lowest quality of optics that can be used without sacrificing the measurement accuracy.

All of the used optical components are standard, readily available from a number of companies. We decided to use optical components with $\lambda / 10$ accuracy and antireflective coatings to reduce back reflections. The lenses used for collimation, focusing and imaging are achromatic doublets, as this type of lens is less sensitive to variation of wavelength, provides smaller focal points and better collimation than single lens.

The beamsplitter is a non-polarizing cube beamsplitter with $50 \mathrm{~mm}$ sides and $\lambda / 8$ accuracy. Cube beamsplitters are useful in a Twyman-Green configuration as they eliminate ghost images that form in plate beamsplitters. They also provide equal path lengths through glass for reference and measurement beams thus eliminating the need for compensator plates.

Collimating and decollimating lens are identical, with a clear aperture of $50 \mathrm{~mm}$. With the beamsplitter that is currently used, this provides a field of view of approximately $48 \mathrm{~mm}$ in diameter, which is more than adequate for gauge block calibration.

\section{PRELIMINARY RESULTS}

Due to the fact that at the time of writing of this paper thermal shielding and environment sensors of the interferometer were not yet installed, only preliminary measurements were made. Since the systems for measurement of temperature of gauge block and the temperature, humidity and pressure of surrounding air are not yet implemented, measurements of length of gauge blocks are not presented. Figure 4 shows an interferogram of a precision mirror with $25 \mathrm{~mm}$ diameter only as a demonstration of optical quality of the new system However, phase shifting measurements of surfaces are not affected with these parameters, so the potential measurement capability of the new interferometer is demonstrated with PSI measurements of gauge block surface, Figure 4, and silicon wafer step height surface, Figure 5.

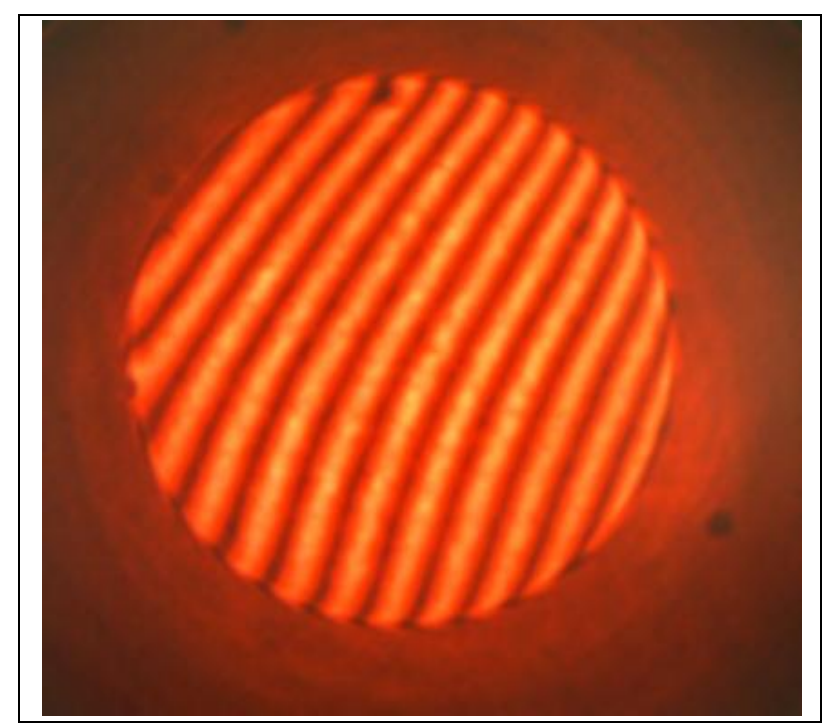

Fig. 5. Interferogram of a mirror with $\lambda / 10$ accuracy 


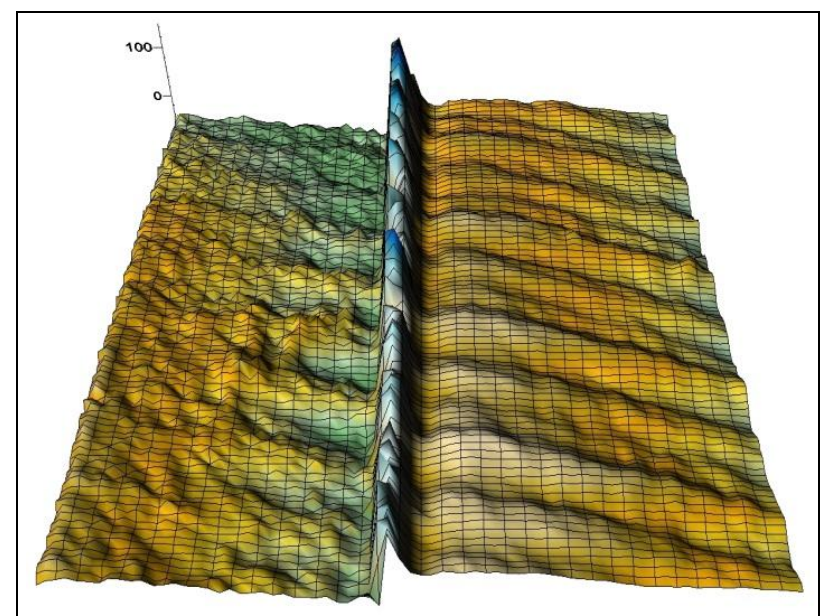

Fig. 6. PSI measurement of a gauge block surface. Platen surface is on the left side, and gauge block surface is to the right, with characteristic polishing marks visible. Total variation of gauge block surface is less than $10 \mathrm{~nm}$.

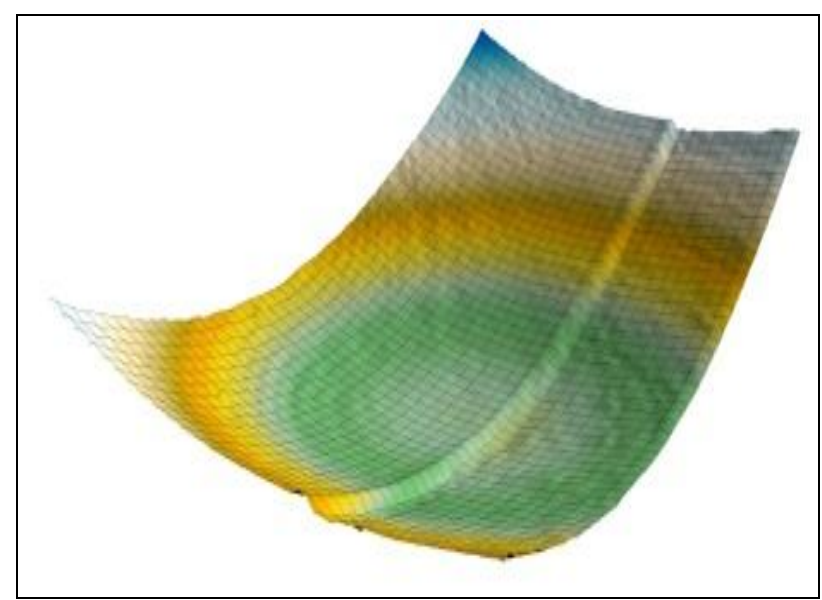

Fig. 7. Silicon wafer step height gauge, with step height of 80 nanometres visible in the centre. Due to its low thickness the entire wafer ( $30 \mathrm{~mm}$ diameter) is deformed by gravity.

\subsection{Comparison with retrofitted Zeiss interferometer}

As an additional demonstration of feasibility of this design, we compared interferograms measured on previously retrofitted Zeiss interferometer with the new system.

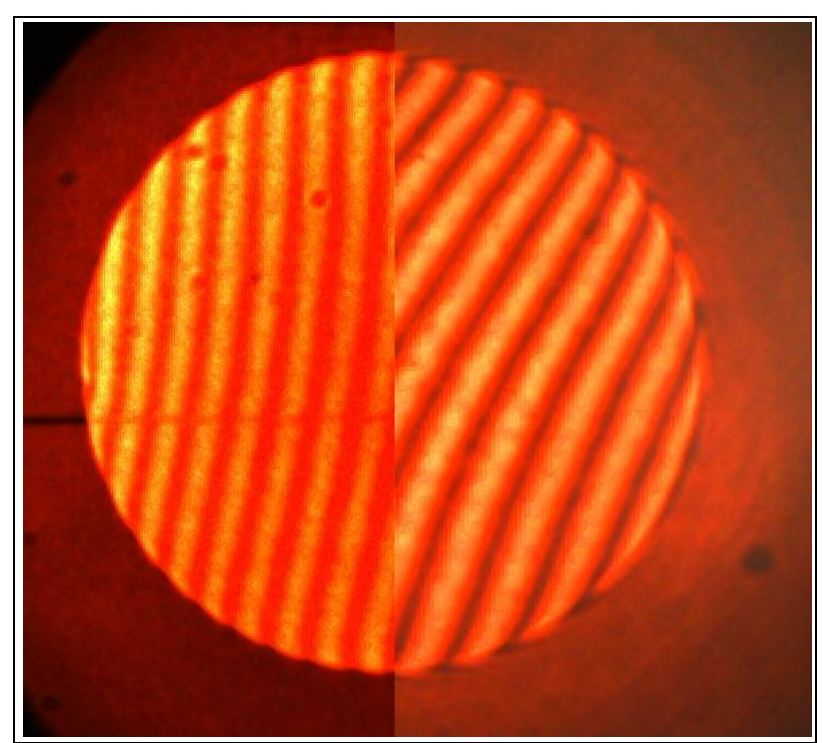

Fig. 8. Comparison of interferograms recorded on retrofitted Zeiss interferometer (left) and new interferometer (right)
Even though optical components that were installed in Zeiss interferometer are several times more accurate, it seems that overall quality of both systems is similar (Figure 6). This can be attributed to large input aperture on the Zeiss interferometer, which is approximately 0.8 $\mathrm{mm}$ in diameter. This is typical for interferometers that have monochromators and are designed for use with broadband light sources, as alignment of monochromators was made visually. Such aperture is significantly larger than the $10 \mu \mathrm{m}$ pinhole used in the new interferometer, and establishes a limit to the optical resolution of the Zeiss interferometer.

\section{CONCLUSION}

A detailed description of theoretical and practical problems concerning gauge block interferometers was given. Based on these considerations, we have presented a design and practical realization of phase shifting gauge block interferometer that can match the accuracy of commercial interferometers at a fraction of their cost. We have demonstrated the measurement capabilities of this system with phase shifting measurements of gauge block surfaces, showing excellent measurement resolution. Comparison with interferograms measured on a Zeiss interferometer indicates that the optical quality is sufficient. This provides certain guarantee that good results will be obtained in measurements of gauge block length. Thermal shielding and environment sensors represent the last major limitation before absolute calibration of gauge blocks can be carried out.

\section{REFERENCES}

[1] Birch, K. P.; Downs, M. J.: Correction to the updated Edlen equation for the refractive index of air, Metrologia v.31, p.315316,1994

[2] ISO 3650:1998, Geometrical Product Specifications (GPS) Length Standards - Gauge blocks

[3] Howick, E. F.; Watkins, L. R.; Tan, S. M.: Automation of a 1960s Hilger gauge block interferometer, 2003, Metrologia 40139 DOI: 10.1088/0026-1394/40/4/301

[4] Decker, J.E.; Bustraan, K.: Updates to the NRC gauge block interferometer, NRC Document No. 42753, 2000

[5] Michalecki, G.: Automatic calibration of gauge blocks measured by optical interferometry, Measurement Science Review, Vol. 1, Sec. 3, 2003., ISSN 1335-8871

[6] Andrew J Lewis et al: Long-term study of gauge block interferometer performance and gauge block stability, 2010 Metrologia 47473 doi:10.1088/0026-1394/47/4/014

[7] Katic, M; Simunovic, V.; Barsic, G.: Modification of a Zeiss Gauge Block Interferometer // The 22nd DAAAM International World Symposium / Vienna, Austria, 2011-11-23/26, p. 805-806.

[8] Katic, M.; Mudronja, V.: Final report on EURAMET.L-K1.2: EURAMET comparison of gauge blocks by interferometry. // Metrologia. 49 (2012) 